\title{
Wave Hop Theory of Long Distance Propagation of Low-Frequency Radio Waves'
}

\author{
Leslie A. Berry \\ Contribution From the Central Radio Propagation Laboratory, National Bureau of Standards, \\ Boulder, Colo.
}

(Received July 15, 1964)

\begin{abstract}
The idea that long radio waves propagating between the earth and ionosphere via discrete hops can be extended far into the shadow region by evaluating a series of complex integrals is exploited in this paper. Illustrative calculations of LF and VLF hops and total fields are shown as a function of distance. The second and higher hops show a pseudoBrewster angle just before the caustic and attenuate like groundwaves in the shadow region.

The form of the series of integrals for an anisotropic ionosphere is given, and a model anisotropic ionosphere varying with height is used in a sample calculation.

Only a small error is caused by writing each hop as the product of an effective ionospheric reflection coefficient and an integral which is a function only of the other characteristics of the path.
\end{abstract}

\section{Introduction}

In low-frequency radio propagation theory, it seems natural to consider the field at a point as the sum of rays or hops: the direct, or groundwave, plus the ray that has been reflected once from the ionosphere, plus the ray that has been reflected twice from the ionosphere (and once from the ground), etc. [see, for example, Johler, 1962]. This point of view is especially convenient for the propagation of pulses, since the different rays arrive at the receiver at different times because of the different length paths they have traveled. The hops are sometimes called time-modes to indicate this separation in time.

More precisely, for a vertical electric dipole source, the vertical electric field is written (with time factor exp $i \omega t$ suppressed) [Wait and Murphy, 1957; Johler, 1962]:

$$
E_{r} \propto E_{0}+\sum_{j=1}^{\infty} \exp \left(-i k_{1} D_{j}\right) D_{j}^{-1} \alpha_{j} F_{j} C_{j}
$$

where $E_{0}$ is the groundwave, $D_{j}$ is the length of the ray path of the $j$ th hop, $\alpha_{j}$ is a convergencedivergence coefficient which corrects for focusing at the ionosphere and defocusing at the earth, $F_{j}$ accounts for the presence of the earth at the receiver and transmitter, and $C_{j}$ is the effective reflection coefficient. For homogeneous isotropic plane earth and ionosphere [Wait and Murphy, 1957],

$$
C_{j}=R_{e}^{j-1} T_{e e}^{j},
$$

where $R_{e}$ and $T_{e e}$ are Fresnel reflection coefficients at the ground and ionosphere, respectively, and

$$
F_{j}=\left(1+R_{e}\right)^{2} .
$$

The form of $C_{j}$ is more complicated if the ionosphere is anisotropic [Johler, 1962], and $F_{j}$ must be modified near grazing incidence on the earth for a spherical model [Wait and Murphy, 1957; Johler, 1962]. Johler [1964] indicates that (1) is adequate within about $2500 \mathrm{~km}$ of the source if $F_{1}$ is modified near the caustic and in the shadow region.

1 This work was begun as part of Advanced Research Projects Agency (ARPA) Project No. 85411 and completed on NBS Project No. 85111. 
Bremmer [1949] showed that the geometric-optic series, (1), could be obtained from the full-wave solution by using the saddle point approximation for certain integrals. A complete and very clear derivation of this connection was given by Wait [1961], who suggested that the field can be found at great distances with a series analogous to (1) if the integrals are evaluated numerically. In section 4, this series of integrals is derived for an inhomogeneous, anisotropic ionosphere. The derivation of the solution for the homogeneous isotropic case is outlined first to give the reader insight into the problem. Some sample calculations presented in section 5 illustrate the behavior of the hops.

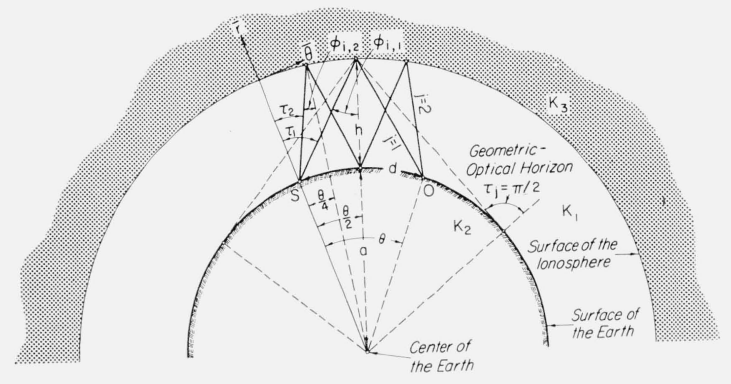

Figure 1. Geometry used.

\section{Homogeneous Isotropic Ionosphere}

The geometry is shown in figure 1 [Johler, 1962]. The center of the earth is the origin of a spherical $(r, \theta, \phi)$ coordinate system. The surface of the earth is $r=a$, and the bottom of the ionosphere is $r=g=a+h$. An elementary vertical dipole source is located at $S(b, 0, \phi), a<b<g$, and the field is to be found at $O(r, \theta, \phi), a<r<g$. The paths taken by the first two hops are shown. The angle of incidence of the $j$ th hop on the earth is $\tau_{j}$, and $\phi_{i, j}$ is its angle of incidence on the ionosphere. The region $\tau_{j}<\frac{\pi}{2}$ is the lit region for the $j$ th hop, $\tau_{j}=\frac{\pi}{2}$ (grazing incidence on the earth) is the caustic, and beyond the caustic is the shadow region.

The media are characterized by their wave numbers. In the air,

$$
k_{1}=\frac{\omega}{c} \eta_{1}
$$

where $\omega=2 \pi f$ is the angular frequency, $c$ is the speed of light, and $\eta_{1}$ is the index of refraction of air. In the earth,

$$
k_{2}=\frac{\omega}{c} \sqrt{\epsilon_{2}-i \frac{\mu_{0} c^{2} \sigma_{2}}{\omega}}
$$

where $\epsilon_{2}$ and $\sigma_{2}$ are permittivity and conductivity of the earth. In the ionosphere,

$$
k_{3}=\frac{\omega}{c} \sqrt{1-i \frac{\omega_{r}}{\omega}}
$$

where $\omega_{r}=\frac{\omega_{N}^{2}}{\nu+i \omega}, \omega_{N}$ is the angular electron plasma frequency, and $\nu$ is the collision frequency.

The vertical electric field is (assuming $r=a=b$ ) [Johler and Berry, 1964]

$$
\begin{aligned}
E_{r} & =\frac{-i L}{k_{1}^{2} a^{4}} \int_{C} \frac{v^{3}}{\cos v \pi} P_{v-1 / 2}(-\cos \theta) \zeta_{a}^{(1)} \zeta_{a}^{(2)}\left(1+R_{e}^{s}\right) \frac{1+p T_{e e}^{s}}{1-p R_{e}^{s} T_{e e}^{s e}} d v \\
& =\int_{C} f(v)\left(1+R_{e}^{s}\right) \frac{1+p T_{e e}^{s}}{1-p R_{e}^{s} T_{e e}^{s}} d v
\end{aligned}
$$


where

$$
L=\frac{\mu_{0} c}{4 \pi} I_{0} l
$$

$I_{0} l$ is the dipole current moment, $C$ is a contour in the fourth quadrant enclosing the poles of the integrand, $P_{v}(x)$ is the Legendre function, $\zeta_{a}^{(K)}=\zeta_{r-1 / 2}^{(K)}\left(k_{1} a\right), \zeta_{v-1 / 2}^{(K)}(x)=\sqrt{\frac{\pi x}{2}} H_{v}^{(K)}(x)$, and $H_{v}^{(K)}(x)$ is a Hankel function.

where

$$
R_{e}^{s}(v)=-\frac{\zeta_{a}^{(1) \prime}-\frac{k_{1}}{k_{2}} R\left(v-\frac{1}{2}\right) \zeta_{a}^{(1)}}{\zeta_{a}^{(2) \prime}-\frac{k_{1}}{k_{2}} R\left(v-\frac{1}{2}\right) \zeta_{a}^{(2)}} \frac{\zeta_{a}^{(2)}}{\zeta_{a}^{(1)}}
$$

where

$$
\begin{gathered}
R\left(v-\frac{1}{2}\right)=\sqrt{\left(\frac{v}{k_{2} a}\right)^{2}-1} . \\
T_{e e}^{s}(v)=-\frac{\zeta_{g}^{(2) \prime}-\frac{k_{1} \zeta_{v-1 / 2}^{(2)}\left(k_{3} g\right)}{k_{3} \zeta_{v-1 / 2}^{(2)}\left(k_{3} g\right)} \zeta_{g}^{(2)}}{\zeta_{g}^{(1) \prime}-\frac{k_{1} \zeta_{v-1 / 2}^{(2)}\left(k_{3} g\right)}{k_{3} \zeta_{v-1 / 2}^{(2)}\left(k_{3} g\right)} \zeta_{g}^{(1)}} \frac{\zeta_{g}^{(1)}}{\zeta_{g}^{(2)}}
\end{gathered}
$$

$$
\zeta_{g}^{(K)}=\zeta_{v-1 / 2}^{(K)}\left(k_{1} g\right) .
$$

$R_{e}^{s}$ and $T_{e e}^{s}$ are called the spherical reflection coefficients since they reduce to the planar Fresnel reflection coefficients when the Debye approximations [Wait, 1960]

$$
\frac{\zeta_{v-1 / 2}^{(M)}(x)}{\zeta_{v-1 / 2}^{(M)}(x)} \cong(-1)^{M+1} i \sqrt{1-\left(\frac{v}{x}\right)^{2}}
$$

are used and $\frac{v}{x}$ is identified as the sine of the angle of incidence:

and

$$
\sin \phi_{i} \sim \frac{v}{k_{1} g},
$$

Finally,

$$
\sin \tau \sim \frac{v}{k_{1} a} .
$$

If

$$
p=\frac{\zeta_{a}^{(1)} \zeta_{g}^{(2)}}{\zeta_{a}^{(2)} \zeta_{g}^{(1)}}
$$

$$
\left|p T_{e e}^{s} R_{e}^{s}\right|<1
$$

on the contour $C$, then $1 /\left(1-p R_{e}^{s} T_{e e}^{s}\right)$ can be expanded in a geometric series. Bremmer [1949] and Wait [1961] assumed that (17) held on some suitable contour, and this has been verified numerically for all cases shown in this paper. Figure 2 shows contours of $\left|p R_{e}^{s} T_{e e}^{s}\right|$ in the complex $v$-plane for a frequency of $100 \mathrm{kc} / \mathrm{s}$ and a particular model of the ionosphere. $\left|p T_{e}^{s} T_{e e}^{e}\right|=1$ on the line of 1's and is less than one above it. So, write

$$
\begin{aligned}
\frac{1+p T_{e e}^{s}}{1-p R_{e}^{s} T_{e e}^{s}} & =\left(1+p T_{e e}^{s}\right)\left(1+\sum_{j=1}^{\infty} p^{j}\left(R_{e}^{s} T_{e e}^{s}\right)^{j}\right) \\
& =1+\left(1+R_{e}^{s}\right) \sum_{j=1}^{\infty}\left(R_{e}^{s}\right)^{j-1}\left(p T_{e e}^{s}\right)^{j} .
\end{aligned}
$$

Substitute (19) into (8) and integrate term by term:

$$
E_{r} \cong \int_{C} f(v)\left(1+R_{e}^{s}\right) d v+\sum_{j=1}^{\infty} \int_{C} f(v)\left(1+R_{e}^{s}\right)^{2}\left(R_{e}^{s}\right)^{j-1}\left(p T_{e e}^{s}\right)^{j} d v
$$




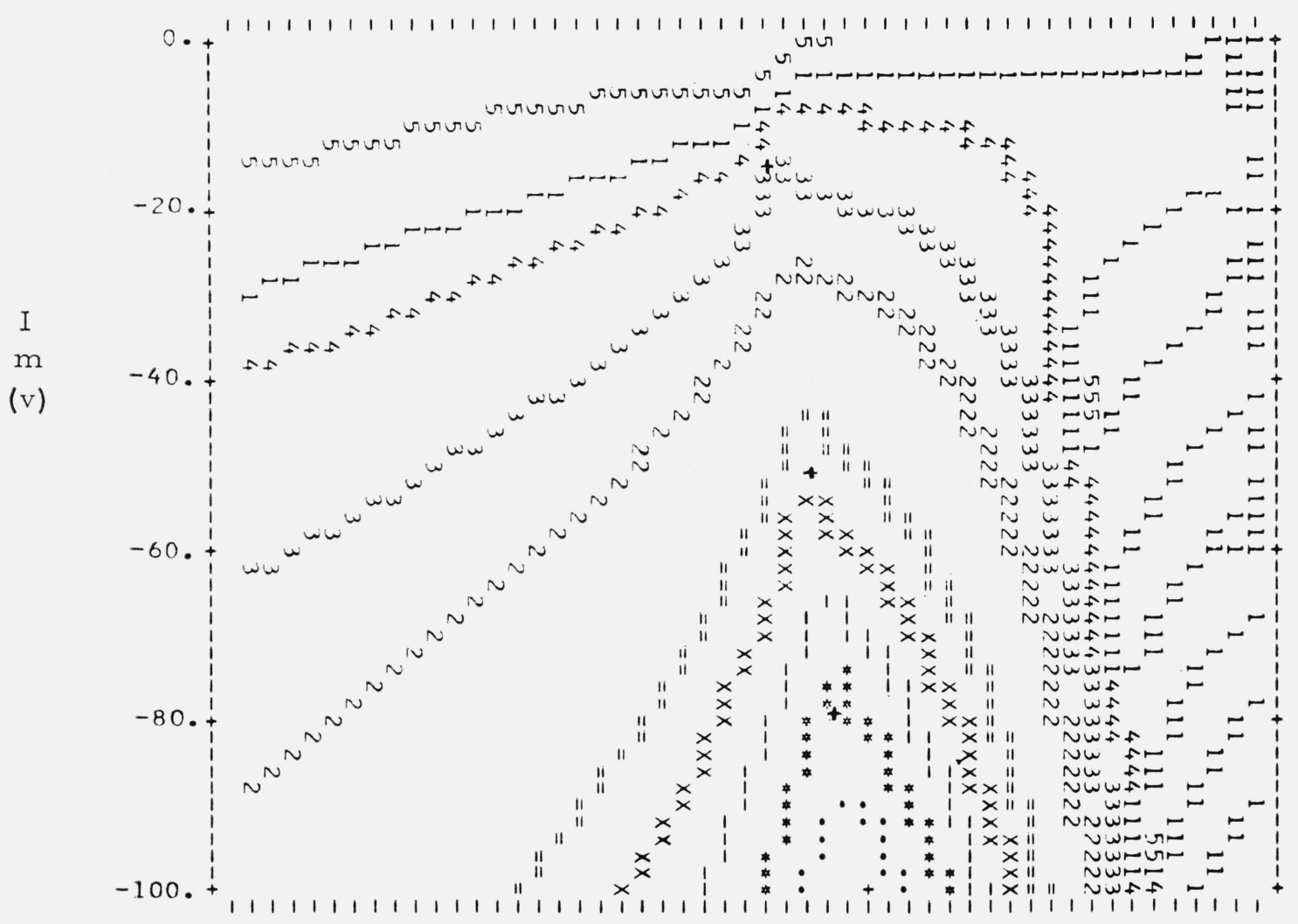

Figure 2. Contours of equal $\left|\mathrm{pT}_{\mathrm{ee}}^{\mathrm{s}} \mathrm{R}_{\mathrm{e}}^{\mathrm{s}}\right|$.

The symbols, in order of decreasing magnitude, are $+, ., \times,=, 2,3,4,1,5$, with $\mid p T_{e e}^{s} R_{e}^{s}=1$ for symbol 1 .

The first integral in (20) is exactly the groundwave [Bremmer, 1949]. If the asymptotic form,

$$
\frac{P_{v-1 / 2}(-\cos \theta)}{\cos v \pi} \cong \sqrt{\frac{2}{\pi\left(v+\frac{1}{2}\right) \sin \theta}} \exp \left[-i\left(v \theta+\frac{\pi}{4}\right)\right]
$$

for $v>>1$, and $\theta$ not near 0 or $\pi$, and the Debye approximations, (13), are used, the integrals in the series in (20) can be evaluated with the saddle point approximation. For more details of the evaluation and a discussion of the physical interpretation of the result, see Wait [1961]. The conclusion is that the $j$ th term of the series in $(20)$ is identified with the $j$ th hop; $\left(1+R_{e}^{s}\right)^{2}$ corresponds to $F_{j}$ in (1); $\left(R_{e}^{s}\right)^{j-1}\left(T_{e e}^{s}\right)^{j}$ corresponds to $C_{j}$, (2); and the other factors in $(20)$ supply the rest of each term in (1). The integrals in the series will be called wave hops since they are full wave solutions that reduce to the ray hops in the limit.

The restrictions on the use of the Debye approximations and the location of the saddle point indicate that the saddle point approximation is inadequate near the caustics $\left(\tau_{j} \sim \frac{\pi}{2}\right)$. Since this paper is primarily concerned with propagation to great distances into the shadow region, the series will be left in the integral form, (20).

The variable of integration $v$ has been related to the angle of incidence $\phi_{i}$ in (14), so the integration is essentially over the angle of incidence. Most of the contribution to the $j$ th integral comes from a small part of the contour corresponding to the geometric angle of incidence. Over this part of the contour, $T_{e e}^{s}(v)$ is a slowly varying function, and the Debye 
approximations for $\zeta_{v-1 / 2}^{(M)}\left(k_{1} g\right)$ are adequate. So, to a good approximation, $T_{e e}^{s}(v)$ can be replaced with the constant Fresnel $T_{e e}$, which is then written outside the integral [Wait, 1961];

$$
E_{r, j} \cong T_{e e}^{j} \int_{c} f(v)\left(1+R_{e}^{s}\right)^{2}\left(R_{e}^{s}\right)^{j-1} p^{j} d v \cong T_{e e}^{j} I_{j}
$$

Each wave hop is the product of two factors: the ionospheric reflection coefficient, which is the most variable factor of a propagation path; and the path integral, $I_{j}$, which is a function of the relatively constant path parameters: ground conductivity, distance, earth curvature, and reflection height. Presumably, reflection coefficients for more sophisticated (e.g., continuously varying) ionospheric models could be used in (22); indeed it is shown in the next section that the solution for the anisotropic case has the same path integral. Tables of the path integrals, if available, could be used with the published tables and graphs of reflection coefficients [Johler, Walters, and Harper, 1960; Wait, 1962; Wait and Walters, 1963a, b, and c; Walters and Wait, 1963] to calculate fields with relatively little effort. A few such results were given by Wait and Conda [1961].

For simplicity, it has been assumed that $r=b=a$, above. If the receiver and/or transmitter are elevated, the integrand of $I_{j}$ is multiplied by $G_{v-1 / 2}\left(k_{1} r\right) \mathrm{G}_{v-1 / 2}\left(k_{1} b\right)$, where

$$
G_{v}(x)=\left(1+R_{e}^{s}\right)^{-1}\left\{\frac{\zeta_{v}^{(1)}(x)}{\zeta_{v}^{(1)}\left(k_{1} a\right)}+\frac{\zeta_{v}^{(2)}(x)}{\zeta_{v}^{(2)}\left(k_{1} a\right)} R_{e}^{s}\right\}
$$

is a height gain function. The integrand of the ground wave is multiplied by

and

$$
\frac{\zeta_{b}^{(2)}}{\zeta_{a}^{(2)}} G_{v-\frac{1}{2}}\left(k_{1} r\right), \text { if } r<b
$$

$$
\frac{\zeta_{r}^{(2)}}{\zeta_{a}^{(2)}} G_{v-\frac{1}{2}}\left(k_{1} b\right), \text { if } r>b .
$$

\section{Homogeneous Anisotropic Ionosphere}

Now, the effect of the earth's magnetic field is considered. Assuming that $\frac{\partial}{\partial \phi}=0$, and the ionosphere is locally planar,

where

$$
E_{r} \cong \int_{c} f(v)\left(1+R_{e}^{s}\right) \frac{\left\{\left(1-p T_{m m} R_{m}^{s}\right)\left(1+p T_{e e}\right)+p^{2} T_{e m} T_{m e} R_{m}^{s}\right\} d v}{\left\{\left(1-p R_{e}^{s} T_{e e}\right)\left(1-p R_{m}^{s} T_{m m}\right)-p^{2} T_{e m} T_{m e} R_{e}^{s} R_{m}^{s}\right\}}
$$

$$
R_{m}^{s}=-\frac{\zeta_{a}^{(1) \prime}-\frac{k_{2}}{k_{1}} R\left(v-\frac{1}{2}\right) \zeta_{a}^{(1)}}{\zeta_{a}^{(2) \prime}-\frac{k_{2}}{k_{1}} R\left(v-\frac{1}{2}\right) \zeta_{a}^{(2)}} \frac{\zeta_{a}^{(2)}}{\zeta_{a}^{(1)}}
$$

The planar anisotropic ionospheric reflection coefficients $T_{e e}, T_{e m}, T_{m e}$, and $T_{m m}$ [Johler and Walters, 1960] correspond to $R_{\|},{ }_{\|} R_{\perp},{ }_{\perp} R_{\|}$, and ${ }_{\perp} R_{\perp}$, respectively, in the notation of Wait [1962] and others. Explicit expressions for the T's are given by Johler and Berry [1964].

Equation (26) was derived by Wait [1963], using impedance boundary conditions, and by Johler and Berry [1964], using other approximate boundary conditions.

To write (26) more concisely, define the matrices

Then

$$
\mathcal{R}_{e}=\left[\begin{array}{cc}
R_{e}^{s} & 0 \\
0 & -1
\end{array}\right], T=\left[\begin{array}{cc}
T_{e e} & T_{e m} \\
T_{m e} & T_{m m}
\end{array}\right] \text {, and } p=p\left[\begin{array}{cc}
1 & 0 \\
0 & -R_{m}^{s}
\end{array}\right] \text {. }
$$

$$
E_{r} \simeq \int_{c} f(v)\left(1+R_{e}^{s}\right) \frac{|I+p T| d v}{\left|I-p R_{e} T\right|}
$$


which looks much like (8). ( $I$ is the identity matrix.) If some norm, \|\| , of $p \mathscr{R}_{e} T$ can be found such that

$$
\left\|p \mathscr{R}_{e} T\right\|<1
$$

on the contour, then $\left|I-p \mathscr{R}_{e} T\right|^{-1}$ can be expanded [Finkbeiner, 1960],

$$
\left|I-p \mathscr{R}_{e} T\right|^{-1}=\left|I+\sum_{j=1}^{\infty}\left(p \mathscr{R}_{e} T\right)^{j}\right|=\left|I+\sum_{j=1}^{\infty} \mathscr{R}_{e}^{j}(p T)^{j}\right|,
$$

so that

$$
\frac{|I-p T|}{\left|I-\mathscr{R}_{e} T\right|}=\left|I-p \mathcal{R}_{e} T\right|^{-1}|I+p T|=\left|I+\left(I+\mathcal{R}_{e}\right) \sum_{j=1}^{\infty} \mathscr{R}_{e}^{j-1}(p T)^{j}\right|
$$

Let

Then, since

$$
(p T)^{j}=p^{j}\left[\begin{array}{ll}
\gamma_{j} & \\
y_{j} & z_{j}
\end{array}\right] .
$$

$$
\begin{gathered}
\left(I+\mathcal{R}_{e}\right)=\left[\begin{array}{ll}
1+R_{e}^{s} & 0 \\
0 & 0
\end{array}\right], \\
\left|I+\left(I+\mathcal{R}_{e}\right) \sum_{j=1}^{\infty} \mathcal{R}_{e}^{j-1}(p T)^{j}\right|=1+\left(1+R_{e}^{s}\right) \sum_{j=1}^{\infty} p^{j}\left(R_{e}^{s}\right)^{j-1} \gamma_{j} .
\end{gathered}
$$

Substitute (35) into (29) and integrate term by term (assuming integration and summation can be interchanged):

$$
E_{r} \cong \int_{c} f(v)\left(1+R_{e}^{s}\right) d v+\sum_{j=1}^{\infty} \int_{c} f(v)\left(1+R_{e}^{s}\right)^{2}\left(R_{e}^{s}\right)^{j-1} p^{j} \gamma_{j} d v
$$

The first integral in (36) is again the groundwave, and the second integral is the same as in (20) except that $T_{e e}^{j}$ has been replaced by $\gamma_{j}$, the effective reflection coefficient. Indeed, if the magnetic field is zero, $T_{e m}=T_{m e}=0$, and $\gamma_{j}=T_{e \varepsilon}^{j}$. Using (33),

and

$$
\begin{gathered}
\gamma_{1}=T_{e e} \\
R_{e}^{s} \gamma_{2}=R_{e}^{s} T_{e e}^{2}-R_{e}^{s} R_{m}^{s} T_{e m} T_{m e}
\end{gathered}
$$

$$
\left(R_{e}^{s}\right)^{2} \gamma_{3}=\left(R_{e}^{s}\right)^{2} T_{e e}^{3}-2\left(R_{e}^{s}\right)^{2} R_{m}^{s} T_{e e} T_{e m} T_{m e}+\left(R_{e}^{s}\right)^{2}\left(R_{m}^{s}\right)^{2} T_{e m} T_{m e} T_{m m} .
$$

These are the effective reflection coefficients $C_{j}$ used by Johler [1961] in the geometric series for anisotropic ionosphere. Equation (33) gives a numerically more satisfactory method of computing $C_{j}$ than the usual formula [Johler, 1961]:

$$
C_{j}=\frac{1}{j ! R_{e}} \frac{d^{j}}{d x^{j}}\left[\frac{1-R_{m} T_{m m} x}{1-\left(R_{e} T_{e e}+R_{m} T_{m m}\right) x+R_{e} R_{m}\left(T_{e e} T_{m m}-T_{e m} T_{m e}\right) x^{2}}\right]_{x=0} .
$$

If the saddle point approximation is used for the integrals in (36), the geometric-optics series is obtained with the same limitations at great ranges as before. As in the isotropic case, $\gamma_{j}$ can be regarded as a constant (with respect to $v$ ) so the wave hops are written $\gamma_{j} I_{j}$, and height gain factors $(23,24$, and 25$)$ can be included in the integrand.

\section{Inhomogeneous Anisotropic Ionosphere}

In the geometric-optic series, it is sometimes assumed that the ionosphere varies along the path and the reflection coefficients are calculated for the local values of the electromagnetic 
parameters. This can be done formally in (36) by writing (compare with Johler [1961])

$$
E_{r, j}=\int_{c} f(v)\left(1+R_{e, 0}^{s}\right)\left(1+R_{e, s}^{s}\right) \prod_{k=1}^{j-1} \mathscr{R}_{e, k} \prod_{k=1}^{j} p_{k} T_{k} d v
$$

where $R_{e, 0}^{s}$ is computed for the ground parameters at the observer, $R_{e, s}^{s}$ for the parameters at the source, and $R_{e, r}^{s}$ and $T_{k}$ are computed for the values at the place where the $j$ th hop is incident on the boundary for the $k$ th time. This is an approximate formula which should be useful when the variation in the path is slow and smooth. Calculations using (39) will be made and reported in the future.

\section{Calculations and Discussion}

The integrals in (20) and (36) can be evaluated numerically using existing subroutines for the Hankel functions [Berry, 1964]. Figures 3, 4, and 5 show the amplitudes of the total field, the groundwave, and the first three wave hops as a function of distance, $d=a \theta$, for $100 \mathrm{kc} / \mathrm{s}$, $30 \mathrm{kc} / \mathrm{s}$, and $10 \mathrm{kc} / \mathrm{s}$. Propagation is over land and for the given model of the sharply bounded ionosphere. The curves are normalized to $I_{0} l=1$, (9). The caustic (grazing incidence on earth) is marked on each hop. One interesting feature of the second hop in figure 3 is the relative minimum at $3100 \mathrm{~km}$ - just before the caustic at $3700 \mathrm{~km}$. This minimum is the pseudo-Brewster angle [Bremmer, 1949] of the spherical ground reflection coefficient, $R_{e}^{s},(10)$. In the third wave hop, $R_{e}^{s}$ is squared, so the minimum in it is sharper. The minima occur at different distances in figures 4 and 5 , and they are not as deep, because the pseudo-Brewster angle is frequency dependent. Figure 6 shows propagation of $100 \mathrm{kc} / \mathrm{s}$ waves over sea water, and the good conductivity nearly eliminates the pseudo-Brewster angle.

At $10 \mathrm{kc} / \mathrm{s}$, figure 5 , the minimum at $2200 \mathrm{~km}$ in the second hop is reflected in the third hop. This is hard to explain with ray theory and shows that the identification of wave hops with rays is not complete.

The first hop does not show the pseudo-Brewster angle because it is not reflected at the ground, but propagates into the shadow region very much like a groundwave. This has been observed experimentally (for example, see Belrose [1964]), and was demonstrated theoretically by Wait [1961]. Wait and Conda [1958] showed how to calculate the effect for the first hop by modifying $F_{1},(1)$ and (3), with a "cutback" factor. All the wave hops include this effect implicitly.

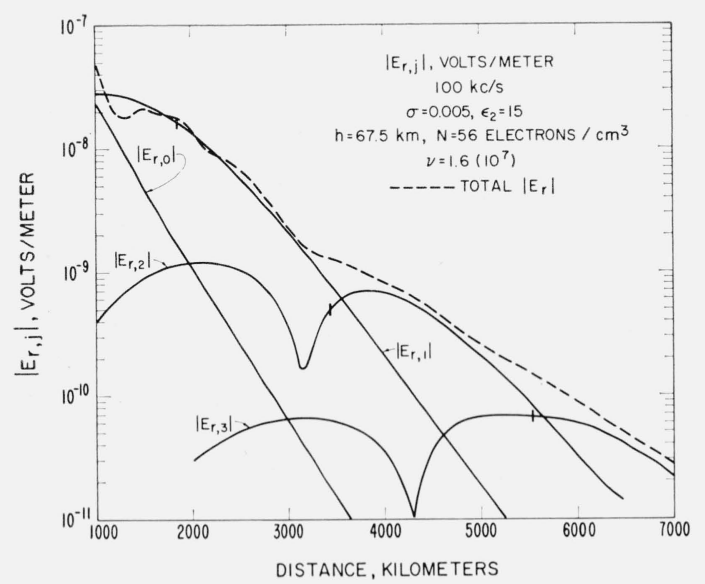

Figure 3. Amplitude of the vertical electric field, and of the individual wave hops, isotropic ionosphere, $\mathrm{f}=100 \mathrm{kc} / \mathrm{s}$, land.

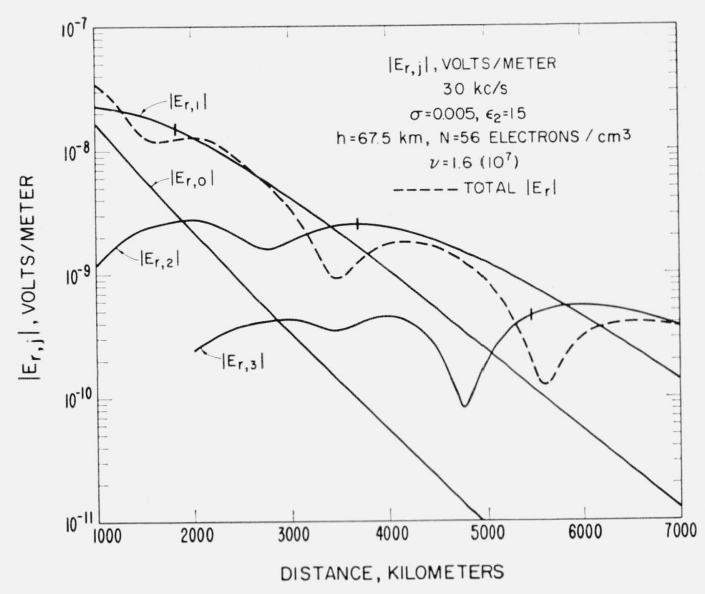

Figure 4. Amplitude of the vertical electric field, and of the individual wave hops, isotropic ionosphere, $\mathrm{f}=30 \mathrm{kc} / \mathrm{s}$, land. 


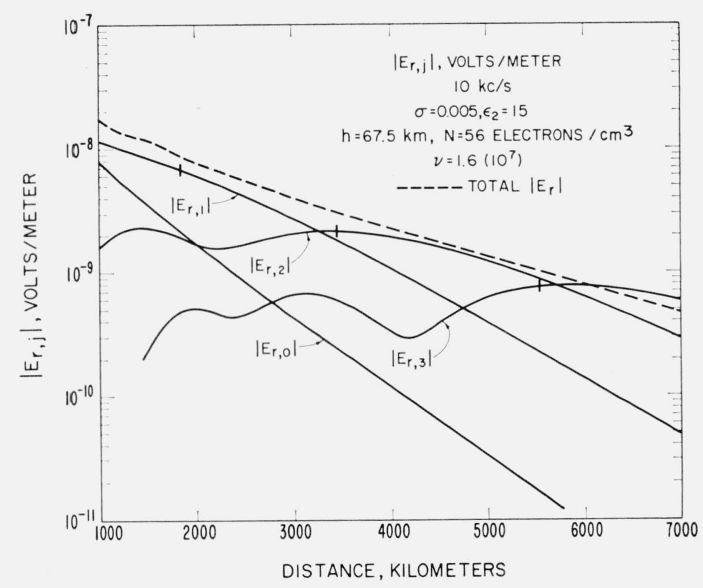

Figure 5. Amplitude of the vertical electric field, and of the individual wave hops, isotropic ionosphere, $\mathrm{f}=10 \mathrm{kc} / \mathrm{s}$, land.

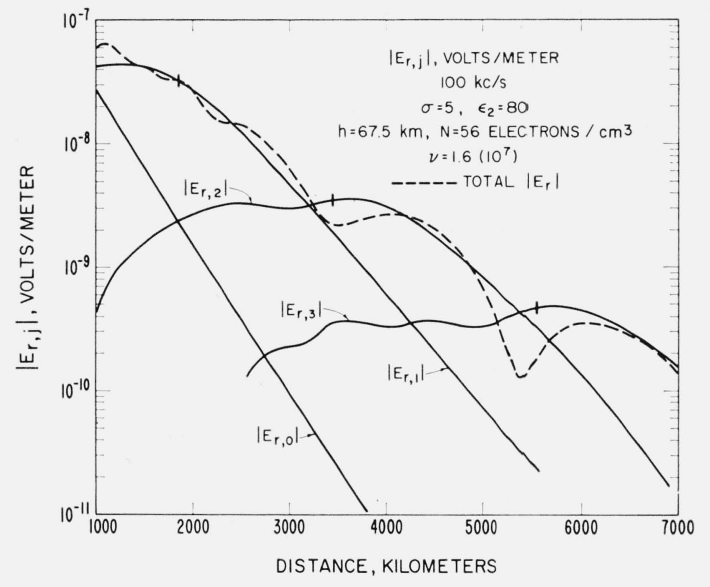

Figure 6. Amplitude of the vertical electric field, and of the individual wave hops, isotropic ionosphere, $\mathrm{f}=100 \mathrm{kc} / \mathrm{s}$, sea.

The total field at $30 \mathrm{kc} / \mathrm{s}$, figure 4 , shows an interference pattern. In mode theory, this is interpreted as interference between the first two modes [Johler and Berry, 1964]. Here, the minimum at $3500 \mathrm{~km}$ is seen to be interference between the first and second hops, and the minimum at $5600 \mathrm{~km}$ is interference between the second and third hops.

For the model chosen, the wave hops are dominant for a considerable distance into their shadow regions. In contrast, geometric-optics cuts off the hop in the shadow region.

Equations (20) and (36) were derived for a sharply bounded, homogeneous model ionosphere. No mathematical justification has been given for replacing the ionospheric reflection coefficients in them with ones for a model that varies with height. There are obvious uncertainties; e.g., what is the effective reflection height (if there is one)? Even so, such replacement seems reasonable, especially for long radio waves.

As an illustration, reflection coefficients for a stratified, planar, anisotropic ionosphere were calculated [Johler, 1962] for the daytime noon electron density profile shown in figure 7 [Pierce, 1963]. These reflection coefficients, $T_{e e}, T_{e m}, T_{m e}$, and $T_{m m}$, were then used in (36) to calculate the propagation of the wave hops into the west over land. The earth's magnetic field vector was assumed to $\operatorname{dip} 60^{\circ}$ with magnitude $0.5 \mathrm{G}$. Figure 8 shows the total field and the first three wave hops. The amplitude of the hops in the lit region shows that this model ionosphere reflects about as well as the model used in figures 3,4 , and 5 . The greater attenuation of the total field is probably due to the lower assumed ionosphere height.

The wave hop theory will be especially useful in the form

$$
E_{r, j} \cong \gamma_{j} I_{j}
$$

where $\gamma_{j}$ is the effective ionospheric reflection coefficient (33) and $I_{j}$ is the path integral (22). To investigate the accuracy of (40), reflection coefficients were calculated for the sharply bounded, isotropic model ionosphere used in figures 3, 4, and 5 using the Fresnel formula [Bremmer, 1949],

$$
T_{e e}=\frac{\cos \phi_{i, j}-\frac{k_{1}}{k_{3}} \sqrt{1-\left(\frac{k_{1}}{k_{3}} \sin \phi_{i, j}\right)^{2}}}{\cos \phi_{i, j}+\frac{k_{1}}{k_{3}} \sqrt{1-\left(\frac{k_{1}}{k_{3}} \sin \phi_{i, j}\right)^{2}}} .
$$




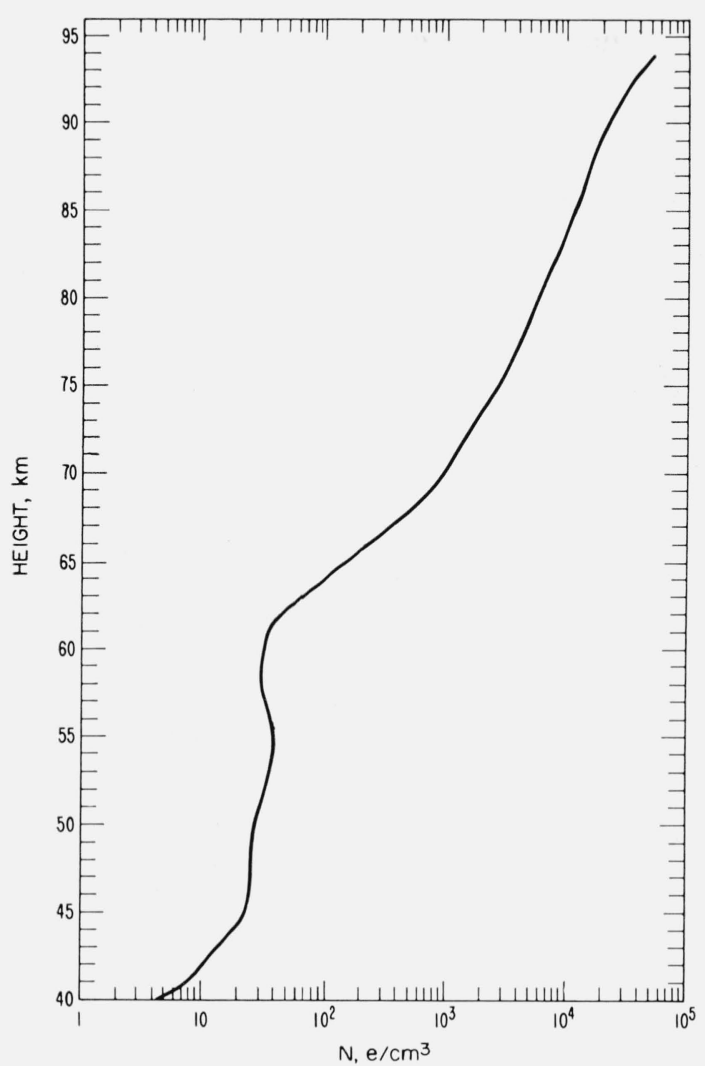

Figure 7. Electron density profile used for calculations shown in figure 8 .

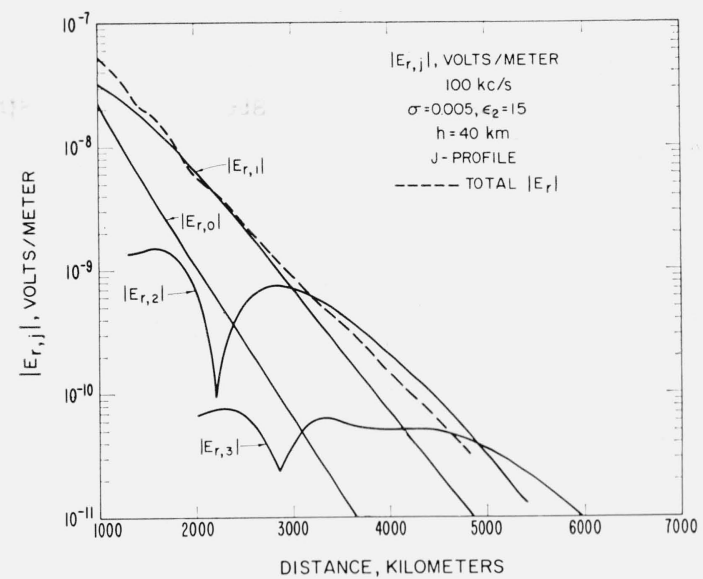

Figure 8. Amplitude of the vertical electric field, and of the individual wave hops, stratified anisotropic ionosphere, $\mathrm{f}=100 \mathrm{kc} / \mathrm{s}$.

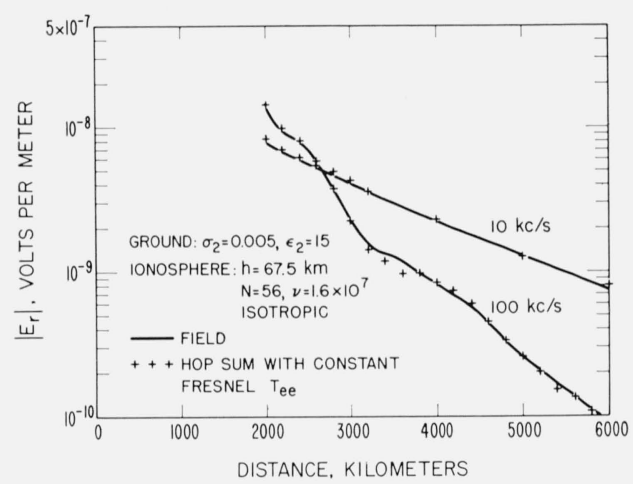

Figure 9. Comparison of field strength at $10 \mathrm{kc} / \mathrm{s}$ and $100 \mathrm{kc} / \mathrm{s}$ with calculations using $(40)$.

The angle of incidence, $\phi_{i, j}$, was calculated geometrically [Johler, 1961]:

$$
\sin \phi_{i, j}=\frac{a \sin \frac{\theta}{2 j}}{\sqrt{2 a g\left(1-\cos \frac{\theta}{2 j}\right)+h^{2}}}
$$

up to the caustic, where $\sin \phi_{i, j}=\frac{a}{g}$ and was held constant at this value in the shadow region.

Figure 9 compares the field at $10 \mathrm{kc} / \mathrm{s}$ and $100 \mathrm{kc} / \mathrm{s}$ with the answers given by (40) using $\gamma_{j}=\mathrm{T}^{j}{ }_{e e}$, (41). The error is negligible for most applications, so the path integrals will be calculated for a range of path parameters to complement the recent extensive reflection coefficient calculations [Johler, Walters, and Harper, 1960; Wait, 1962; Wait and Walters, 1963 a, b, c; Walters and Wait, 1963].

\section{Conclusions}

The wave hop theory of propagation extends the useful notion of geometric-optics to great distances - deep into the shadow region. The theory lends itself readily to physical interpretation, especially in the propagation of low-frequency pulses. 
The sample calculations show the characteristics of the hops as a function of distance. The second and higher hops have a relative minimum just before their caustics caused by the pseudo-Brewster angle of the spherical ground reflection coefficient. In the shadow region the hops propagate much like a groundwave.

Each wave hop can be written as the product of an ionospheric reflection coefficient and a path integral. The path integrals are the natural complements of the ionospheric reflection coefficients since they have the same form for each model of the ionosphere. They will be calculated for a range of parameters in the frequency range $10 \mathrm{kc} / \mathrm{s}$ to $100 \mathrm{kc} / \mathrm{s}$ and will be made available in the near future.

The author is greatly indebted to J. R. Johler for initiating the research reported here and for guidance in its development.

\section{References}

Belrose, J. S. (1964), The oblique reflection of CW low-frequency radio waves from the ionosphere, Propagation of radio waves at frequencies below $300 \mathrm{kc} / \mathrm{s}$, AGARDograph $\mathbf{7 4}$ (Pergamon Press, New York, N.Y.).

Berry, L. A. (1964), Computation of Hankel functions, NBS Tech. Note No. 216.

Bremmer, H. (1949), Terrestrial radio waves (Elsevier Publishing Co., New York, N.Y.).

Finkbeiner, D. T. (1960), Matrices and linear transformations II (W. H. Freeman \& Co., San Francisco, Calif.).

Johler, J. R. (1961), On the analysis of LF ionospheric radio propagation phenomena, J. Res. NBS 65D, (Radio Prop.), No. 5, 507-529.

Johler, J. R. (1962), Propagation of low-frequency radio signal, Proc. IRE 50, No. 4, 404-427.

Johler, J. R. (1964), Concerning limitations and further corrections to geometric-optical theory for LF/VLF propagation between the ionosphere and the ground, Radio Sci. J. Res. NBS/USNC-URSI 68D, No. 1, $67-78$.

Johler J. R., and L. A. Berry (1964), A complete mode sum for LF, VLF, ELF terrestrial radio waves, NBS Monograph No 78 .

Johler, J. R., and L. C. Walters (1960), On the theory of low- and very-low-radio frequency waves from the ionosphere, J. Res. NBS 64D, (Radio Prop.), No. 3, 269-285.

Johler, J. R., L. C. Walters, and J. D. Harper, Jr. (1960), Low-and very-low-radio frequency, model ionosphere reflection coefficients, NBS Tech. Note No. 69.

Pierce, E. T. (1963), Collisional detachment and the formation of an ionosperic C region, J. Res. NBS, 67D, (Radio Prop.), No. 5, 525-532.

Wait, J. R. (1960), Terrestrial propagation of very low-frequency radio waves, J. Res. NBS 64D, (Radio Prop.), No. 2, 153-204.

Wait, J. R. (1961), A diffraction theory for LF sky-wave propagation, J. Geophy. Res. 66, No. 6, 1713-1724.

Wait, J. R. (1962), Electromagnetic waves in stratified media (Pergamon Press Inc., New York, N.Y.).

Wait, J. R. (1963), The mode theory of VLF radio propagation for a spherical earth and a concentric anisotropic ionosphere, Can. J. Phys. 41, 299-305.

Wait, J. R., and A. M. Conda (1958), Pattern of an antenna on a curved lossy surface, IRE Trans. Ant. Prop. AP-6, No. 4, 348-359.

Wait, J. R., and A. M. Conda (1961), A diffraction theory of LF sky-wave propagation-an additional note, J. Geophy. Res. 66, No. 6, 1725-1729.

Wait, J. R., and A. Murphy (1957), The geometric-optics of VLF sky-wave propagation, Proc. IRE 45, No. 6, 754-760.

Wait, J. R., and L. C. Walters (1963a), Reflection of VLF radio waves from an inhomogeneous ionosphere. Part I. Exponentially varying isotropic model, J. Res. NBS 6rD (Radio Prop.), No. 3, 361-367.

Wait, J. R., and L. C. Walters (1963b), Reflection of VLF radio waves from an inhomogeneous ionosphere. Part II. Perturbed exponential model, J. Res. NBS 69D (Radio Prop.), No. 5, 519-523.

Wait, J. R., and L. C. Walters (1963c), Reflection of VLF radio waves from an inhomogeneous ionosphere. Part III. Exponential model with hyperbolic transition, J. Res. NBS 6zD (Radio Prop.), No. 6, 747-752.

Walters, L. C., and J. R. Wait (1963), Numerical calculations for reflection of electromagnetic waves from a lossy magneto plasma, NBS Tech. Note No. 205.

(Paper 68D12-432) 